\title{
Zehn Jahre Governance der Eurozone: ökonomische Bilanz und institutionelle Dynamiken jenseits der Vertragsrevisionen
}

\author{
Daniela Schwarzer*
}

Die Auswirkungen der globalen Wirtschafts- und Finanzkrise machen vor der Europäischen Währungsunion (EWU) ${ }^{1}$ nicht halt: Zum zehnten Geburtstag des Euro ist die EWU erstmals in eine Rezession gerutscht. Massive Arbeitsplatzverluste und Druck auf die Staatsfinanzen der Mitgliedstaaten sind in der Folge zu erwarten. Da die Krise nicht die gesamte Währungsunion mit gleicher Härte und gleichen Konsequenzen trifft, dürften wachsende ökonomische Divergenzen und divergierende politische Präferenzen die EWU und ihre Governance-Mechanismen vor eine Bewährungsprobe stellen.

Ökonomische Entwicklungen und die ersten Erfahrungen mit einem historisch einmaligen institutionellen Gefüge haben bereits in den ersten zehn Jahren seit Beginn der Währungsunion zu politischen Dynamiken und zu expliziten wie schleichenden Anpassungen ihres institutionellen Rahmens geführt. Dieser Beitrag bilanziert erstens die ökonomischen Entwicklungen der ersten Dekade der Europäischen Währungsunion und untersucht zweitens die Vertiefungs- und Erweiterungsdynamiken in der Eurozone. ${ }^{2}$ Dabei werden die Auswirkungen der aktuellen Finanzmarkt- und Wirtschaftskrise besonders berücksichtigt, die in der EWU eine intensivierte Governance-Diskussion ausgelöst hat.

\section{Ökonomische Bilanz nach zehn Jahren}

\section{Wachstum, Inflation und Beschäftigungsentwicklung 1999-2008}

Die Europäische Zentralbank (EZB) hat für die ersten Jahre des Euro eine solide geldpolitische Bilanz aufzuweisen. Die Inflation war mit im Durchschnitt 2,0 Prozent (1999-2007) im historischen und internationalen Vergleich in den ersten neun Jahren seit Einführung des Euro niedrig (Inflation in den USA im Vergleichszeitraum 2,7 Prozent). Da die EZB an den Märkten rasch an Glaubwürdigkeit gewann und die Inflationserwartungen sanken, pendelten sich die Zinsen an den Geld- und Kapitalmärkten der Eurozone auf niedrigem Niveau ein. Mit Preissteigerungsraten von geschätzten 3,4 Prozent wurde 2008 allerdings zum Jahr der Inflation, ${ }^{3}$ vor allem wegen des Preisauftriebs im Energie- und Nahrungsmittelsektor und aufgrund steigender Löhne. Dennoch haben sich ursprüngliche Befürchtungen, die EWU könnte eine Inflationsgemeinschaft werden, bislang als unbegründet erwiesen. Bereits für

* Dr. Daniela Schwarzer, Leiterin der Forschungsgruppe EU-Integration, Stiftung Wissenschaft und Politik, Berlin.

1 Die Währungsunion umfasst seit 01.01.2009 16 EU-Staaten: Belgien, Deutschland, Finnland, Frankreich, Griechenland, Irland, Italien, Luxemburg, Malta, die Niederlande, Österreich, Portugal, Slowenien, die Slowakei, Spanien und Zypern.

2 Der Artikel konzentriert sich auf interne Governance-Mechanismen im makroökonomischen Bereich. Für eine Diskussion der Dynamiken im Bereich der Lissabon-Agenda und der Außenrepräsentation der Eurozone siehe Paul van den Noord/Björn Döhring/Sven Langedijk/João Nogueira Martins/Lucio Pench/Heliodoro Temprano-Arroyo/Michael Thiel: The Evolution of Economic Governance in EMU, in: Europäische Kommission (Hrsg.): Economic Papers 328/2008.

3 Commerzbank AG: konjunktur, zinsen und wechselkurse - international. Economic Research. Jahresausgabe, Dezember 2008/Januar 2009, S. 6. 
das Jahr 2009 wird ein Absinken der Inflationsrate auf deutlich unter 2 Prozent prognostiziert. Somit stellt sich eher die Frage, ob die EWU in eine Deflation rutschen könnte.

Die Wachstumsbilanz für die Eurozone fiel auch schon vor der aktuellen Krise und nachfolgenden Rezession nur mittelmäßig aus. Von 1999 bis 2007 wuchs das Bruttoinlandsprodukt (BIP) der EWU-Staaten mit lediglich 2,2 Prozent (im Vergleichszeitraum waren es in den USA 2,8 Prozent, in der Europäischen Union insgesamt 2,5 Prozent). Dabei waren die Jahre 2002 (0,9 Prozent BIP-Wachstum in der Eurozone) und 2003 (0,8 Prozent) besonders schwach ausgeprägt. Auch für das Jahr 2008 wird das BIP-Wachstum in der Eurozone auf 0,9 Prozent geschätzt. Für das Jahr 2009 sind die Prognosen noch deutlich schlechter. Die Arbeitslosenquote, die sich bei gut 7 Prozent im Eurozonendurchschnitt eingependelt hatte, dürfte aufgrund des Konjunktureinbruchs 2009 auf deutlich über 8 Prozent ansteigen und sich frühestens 2010 erholen. ${ }^{4}$

\section{Wirtschaftliche Integration unter den Bedingungen des Euro}

Die Erwartung, dass der Euro die wirtschaftliche Integration innerhalb der EWU weiter verstärken würde, hat sich bestätigt: Der Anteil des Intra-EWU-Handels stieg von 27 Prozent im Jahr 1999 auf 32 Prozent im Jahr 2006. Neben anderen Faktoren (Liberalisierungen, Harmonisierung von Finanzmarktregeln, Fortschritte in der Informations- und Kommunikationstechnologie, etc.) trug der Euro zur weiteren Integration der europäischen Finanzmärkte bei, wenngleich hier in einigen Bereichen noch Schritte ausstehen. ${ }^{5}$ Auch im Bereich der Güter- und Dienstleistungsmärkte besteht weiterer Integrationsbedarf, wenn dem gemeinsamen Währungsraum auch eine vollintegrierte europäische Volkswirtschaft gegenüber stehen soll. So lange dies nicht der Fall ist, besteht die Gefahr, dass unzureichend funktionierende grenzüberschreitende Marktmechanismen asymmetrische Schocks nur unzureichend abfangen und zu einer Verstärkung regionaler Konjunkturzyklen führen. ${ }^{6}$

Vorteilhaft erwies sich in Zeiten der globalen Finanzmarktkrise der Wegfall der Wechselkurse in der EWU. Deren Mitgliedstaaten profitieren davon, dass der Konjunktureinbruch nicht durch Wechselkursschwankungen unter den an der EWU teilnehmenden Währungen verschärft wird. Wie schon bei der Finanzmarktkrise infolge des Platzens der NewEconomy-Blase im Jahr 2003 bleiben den teilnehmenden Volkswirtschaften so zusätzliche Belastungen für das Wachstum erspart. Auch und insbesondere Deutschland profitiert davon: Wäre die D-Mark noch als Währung im Einsatz, wäre sie in der aktuellen Krise sicherlich erheblichen Aufwertungsrisiken ausgesetzt, da große Mengen Kapital in die größte Volkswirtschaft mit dem tiefsten Finanzmarkt und einer als am verlässlichsten angesehenen Stabilitätsorientierung der Europäischen Union geströmt wären. Dies hätte Deutschlands Exportfähigkeit massiv belastet und bei einem Offenheitsgrad von rund 40 Prozent des BIP das Wachstum erheblich gebremst. ${ }^{7}$

\section{Divergenzen in der EWU und daraus resultierendes Spannungspotenzial}

Hinter der weitgehend akzeptablen ,Kulisse' der Durchschnittswerte für die Inflation, Zinsen und - mit Abstrichen - für das Wachstum haben sich allerdings beträchtliche Ungleichgewichte und Divergenzen zwischen den teilnehmenden Mitgliedstaaten herausgebil-

4 Ebenda.

5 Werner Becker: Der Euro wird zehn. Den Kinderschuhen entwachsen, in: Deutsche Bank Research (Hrsg.): EU-Monitor 57/2008, S. 10.

6 Siehe Sebastian Dullien/Daniela Schwarzer: Eurozone unter Hochspannung, SWP-Aktuell 21/2005.

7 Der Anteil von Exporten in Nicht-EWU-Staaten liegt heute für Deutschland bei 24,3 Prozent des BIP. 
det. Ökonomen sehen diese teilweise als so gravierend an, dass sie ihres Erachtens die Stabilität der Währungsunion gefährden könnten.

Ein wichtiger Indikator dafür sind die Leistungsbilanzen. Deutschland oder die Niederlande verzeichnen beispielsweise deutliche Überschüsse gegenüber den EU-Partnern. Deutschland entwickelte aus einem Leistungsbilanzdefizit 1999 bis 2007 im Jahr 2008 ein Plus von 6,7 Prozent des BIP. Länder wie Spanien, Portugal oder Italien wiesen hingegen auffällige Leistungsbilanzdefizite auf. Auch in Frankreich ist aus einem Überschuss zu Beginn der EWU ein Außenhandelsdefizit von 2,5 Prozent des BIP entstanden. Diese Leistungsbilanzdefizite deuten auf einen Verlust ihrer Wettbewerbsfähigkeit hin. Realwirtschaftlich spielen sie eine Rolle, da sie mit dem Aufbau einer externen Verschuldungsposition einhergehen. Wenn diese Schulden bedient werden müssen, kann dies die Binnennachfrage erheblich belasten.

In den Leistungsbilanzen schlägt sich die unterschiedliche Entwicklung der Lohnstückkosten seit 1999 nieder. In Italien und Spanien stiegen diese um rund 20 Prozent, in Frankreich um 15 Prozent. Einige Länder haben also aufgrund permanent überhöhter Lohnabschlüsse an Wettbewerbsfähigkeit eingebüßt. ${ }^{8}$ Diese Entwicklung ist nicht ursächlich mit der Einführung des Euro verbunden. Die Auswirkungen überhöhter Lohnabschlüsse sind aber für die betroffenen Volkswirtschaften unter den Bedingungen der EWU nicht mehr so abzufedern, wie dies bei nationalen Währungen der Fall war: Mit der Einführung des Euro ist die Möglichkeit weggefallen, die nationale Währung abzuwerten, um international wieder wettbewerbsfähig zu werden. Aus den exzessiven Lohnzuwächsen resultierten in einigen Ländern wie beispielsweise Griechenland, Portugal und auch Italien erhöhte Preissteigerungsraten und ein Verlust an Wettbewerbsfähigkeit, der sich nur durch eine Senkung oder zumindest Stabilisierung der Lohnstückkosten wieder korrigieren ließe. Dies ist allerdings ein ebenso langwieriger, wie angesichts der bestehenden Lohnverhandlungssysteme schwer durchsetzbarer Prozess.

In Deutschland blieben die Lohnstückkosten aufgrund von Lohnzurückhaltung, Restrukturierung und Reformen stabil. Die in der Folge niedrigere Inflationsrate als im EWUDurchschnitt führte zu einer realen Abwertung, so dass Deutschland gegenüber wichtigen Handelspartnern und Konkurrenten in und außerhalb der EWU deutlich an Wettbewerbsfähigkeit gewann und den temporär durch die Wiedervereinigung verursachten relativen Verlust an Wettbewerbsfähigkeit ausgleichen konnte.

Auch während des kurzen Wirtschaftsaufschwungs 2007/2008 divergierten die Entwicklungen bei Wettbewerbsfähigkeit und Inflation in der Eurozone deutlich. In der Rezession dürfte diese Tendenz noch klarer zutage treten. Auch ohne neue Beitritte zur Eurozone wird erwartet, dass die Divergenzen bei Wachstum, Inflation und Beschäftigungsentwicklung innerhalb der EWU in den kommenden Jahren zunehmen. ${ }^{9}$ Dies kann dazu führen, dass die ,One-Size-Fits-All'-Geldpolitik der EZB, die sich an Durchschnittswerten orientieren muss, in einigen Ländern oder Regionen Konjunkturzyklen verstärkt und verlängert. Längere Abschwungphasen können negative Folgen für die Investitionsbereitschaft der Unternehmen, die Beschäftigungsentwicklung und das Wirtschaftswachstum haben. ${ }^{10}$

Auch die Haushaltslagen werden zwischen den einzelnen EWU-Staaten insbesondere nach den unerwarteten Milliardenausgaben für Konjunkturprogramme zur Belebung der

8 Sebastian Dullien/Ulrich Fritsche: Anhaltende Divergenz bei Inflations- und Lohnentwicklung in der Eurozone: Gefahr für die Währungsunion?, in: Vierteljahreshefte zur Wirtschaftsforschung 4/2007, S. 56-76.

9 BNP Paribas: Eurozone: Internal Imbalance. Market Economics, April 2008.

10 Für eine ausführliche Fassung dieses Arguments siehe Dullien/Schwarzer: Eurozone unter Hochspannung, 2005. 
Wirtschaft und für die Stützung des Finanzsektors stark divergieren. Diese Divergenzen sind in ihrer potenziellen Wirkung nicht zu unterschätzen. ${ }^{11}$ Der in einigen Ländern massive Druck auf die Staatsfinanzen und die in der Folge erwarteten hohen Defizitquoten könnten zu erneuten Auseinandersetzungen über das fiskalpolitische Regelwerk der EWU führen.

Unsolide Staatsfinanzen oder auch mögliche erneute Austrittsdiskussionen in einem oder mehreren EWU-Staaten könnten zudem spekulative Devisenmarktreaktionen auslösen, die zu einer Erhöhung der Zinsen auf Staatsanleihen führen. Damit könnte die Refinanzierung fälliger Schuldtitel und die des laufenden Haushaltsdefizits unmöglich werden. Bereits heute, auch ohne ernst zu nehmende EU-Austrittsdiskussionen auf politischer Ebene, bewerten die Märkte erstmals seit Einführung des Euro die Mitgliedstaaten individueller. Das zeigen die Zinsspreads, die zwischen den Staatsanleihen einiger Länder um über 2 Prozentpunkte variieren (3,03 Prozent Deutschland, 4,45 Prozent Italien, 5,73 Prozent Griechenland). ${ }^{12}$

\section{Außenwert}

Der Außenwert des Euro hat in der zehnjährigen Geschichte der Währungsunion wiederholt für politische Diskussionen gesorgt. Nach der Einführung der neuen Währung am 1. Januar 1999 fiel der Wert des Euro gegenüber den wichtigsten Währungen zunächst deutlich ab und erreichte etwa gegenüber dem US-Dollar im Juni 2001 seinen Tiefststand von 0,85 US-Dollar. Dies war zum einen auf Zinsdifferenziale zwischen Euro und US-Dollar wie auch auf höhere Wachstumserwartungen in den USA angesichts des New-Economy-Booms zurückzuführen. In der EWU entwickelte sich eine Diskussion über die Frage, ob es dem Euro nicht gelungen sei, ausreichend Vertrauen der Anleger zu gewinnen, und ob Interventionen mit dem Ziel seiner Stützung sinnvoll seien.

Mitte des Jahres 2002 begann sich der Euro gegenüber dem US-Dollar zu erholen, als die Dotcom-Blase platzte, sich die Zinsdifferenziale umkehrten und sich das amerikanische Leistungsbilanzdefizit immer weiter ausdehnte. ${ }^{13}$ Gleichzeitig kam die EWU nach der Schwächephase 2002/2003 auf einen deutlicheren Wachstumskurs zurück. Der Euro setzte auch angesichts des Niedergangs der US-Wirtschaft - ab 2005 zu einem Höhenflug an. Zwar zeugte dies einerseits von dem Glaubwürdigkeitsgewinn der europäischen Währung. Andererseits aber setzte der hohe Außenwert des Euro die europäische Exportwirtschaft klar unter Druck und ließ die unterschiedliche Wettbewerbsfähigkeit der Wirtschaften der teilnehmenden Staaten deutlich werden.

Die Diskussionen über Divergenzen und Spannungen in der Eurozone haben sich auch in der aktuellen Krise nicht in einem offensichtlichen Glaubwürdigkeitsverlust des Euro niedergeschlagen - auch wenn der gefallene Außenwert des Euro gegenüber US-Dollar und japanischem Yen im zweiten Halbjahr 2008 auffällig war. Von seinem bisherigen Höchststand am 15. Juli 2008 ( 1 Euro = 1,59 US-Dollar) verlor der Euro gegenüber dem US-Dollar bis Ende November 2008 fast 20 Prozent an Wert, bevor er sich temporär wieder etwas erholte. Die Gründe für den Kursverlust sind vielfältig. Kapitalabflüsse in den Dollar- und den Yen-Raum werden unter anderem darauf zurückgeführt, dass Investoren die Liquidität der

11 Siehe hierzu beispielsweise Joachim Fels: Euro Wreckage? A Remix, in: Morgan Stanley (Hrsg.): Global Economic Forum, 07.11.2008.

12 Thomson Reuters: 10 year government bond spreads am 20.01.2009, abrufbar unter: www.markets.ft.com (letzter Zugriff: 20.01.2009).

13 Für eine ausführlichere Diskussion der Entwicklungen bis 2008 siehe: Europäische Kommission: Euro@ten. Successes and challenges after ten years of Economic and Monetary Union, in: European Economy 2/2008, S. 41-42. 
Finanzmärkte und die Sicherheit öffentlicher Anleihen für höher halten, als dies im parzellierten europäischen Anleihenmarkt der Fall ist. Denn hier gibt es keine von den EU-Staaten gemeinsam abgesicherten Euro-Bonds, sondern nationale Märkte für öffentliche Anleihen unterschiedlicher Bonität. ${ }^{14}$ Hinzu kam ein Wegbrechen des sogenannten Carry-Trade ${ }^{15}$ gegenüber dem Euro. Bei dieser Anlagestrategie leihen Spekulanten in Japan und den USA große Summen zu niedrigen Zinsen und legen sie für höhere Zinsen in der Eurozone an, was den Eurokurs über die realwirtschaftlichen Grundlagen hinaus in die Höhe getrieben hatte. Darüber hinaus gibt es auch Zweifel, inwieweit die Europäische Union in der Lage ist, geeint, entschieden und rasch genug auf die Finanzmarkt- und Wirtschaftskrise zu reagieren.

\section{Neue Anziehungskraft auf die Nicht-Mitglieder}

\section{Umdenken bei den politischen Opt-Outs}

Trotz dieser möglichen Zweifel ist die Attraktivität des Euro für noch nicht teilnehmende EU-Mitgliedstaaten sowie Island stark gestiegen. Dänemark und Großbritannien, die sich im Vertrag von Maastricht ein Opt-Out aus der Währungsunion gesichert hatten, debattieren gegenwärtig ernsthaft über einen Beitritt. Dänemark erlebte im Oktober 2008 eine spekulative Attacke auf die Krone, die zu einer Abwertung der Währung und einer Erhöhung der Zentralbankzinsen auf 5,5 Prozent führte (zuvor hatte die dänische Zentralbank sich weitgehend am EZB-Zins orientiert und am Europäischen Wechselkursmechanismus (EWS II) teilgenommen). Seither hat sich aufgrund der Marktturbulenzen und der Sorge um die Stabilität des nationalen Bankensektors die öffentliche Meinung so gewandelt, dass Premierminister Anders Fogh Rasmussen ein baldiges Beitrittsreferendum in Erwägung zieht. Auch in Schweden, das aus politischen Gründen dafür optiert hat, die Beitrittskriterien im EG-Vertrag nicht zu erfüllen, entwickeln sich Diskussionen um einen Kurswechsel in Bezug auf den Euro. Weitere Marktturbulenzen und Spekulationen gegen die Währungen könnten dazu führen, dass die Kosten-Nutzen-Abwägung hinsichtlich eines EWU-Beitritts sich so verändert, dass ein Beitritt favorisiert wird.

\section{Dynamiken in Mittel- und Osteuropa}

Nachdem der Beitritt zur Eurozone noch im vergangenen Jahr in Polen grundsätzlich infrage gestellt wurde und, dem Beispiel Schwedens folgend, ein EG-vertragswidriges Referendum ${ }^{16}$ dazu durchgeführt werden sollte - strebt die Regierung Tusk nun eine Mitgliedschaft bis zum Jahr 2012 an und will bereits im Juni 2009 Mitglied im EWS II werden. ${ }^{17}$ Eine deutliche Abwertung des Złoty gegenüber dem Euro hatte die Sorge vor weiterem Druck auf die polnische Währung und den polnischen Finanzmarkt wachsen lassen.

Auch die Tschechische Republik und Ungarn haben jüngst ihren EWU-skeptischen Kurs aufgegeben. Ähnlich wie die baltischen Staaten, die mit einem Beitritt im Jahr 2011 liebäugeln, treffen sie Vorbereitungen für eine baldige Konvergenzprüfung, da die Vorteile einer EWU-Mitgliedschaft plötzlich höher als der Verlust der nationalen Währung eingeschätzt

14 Daniel Gros/Stefano Micossi: A call for a European Financial Stability Fund, in: Centre for European Policy (Hrsg.): CEPS Commentary, 30.10.2008.

15 Heiner Flassbeck: Carry Trade - Der Devisenmarkt führt die Ökonomie ad absurdum und die Ökonomen schweigen, in: Financial Times Deutschland, 08.02.2008.

16 Der EG-Vertrag sieht eine automatische Mitgliedschaft in der EWU vor, sobald die Konvergenzkriterien erfüllt werden. Somit ist die Mitgliedschaft in der EWU Teil des acquis communautaire, zu dessen Übernahme sich beitretende Staaten verpflichten.

17 Philippa Runner: Financial crisis builds Polish euro-entry momentum, in: euobserver.com, 28.10.2008. 
werden. Unter dem Eindruck der aktuellen Finanzmarkt- und Wirtschaftskrise werden die Vorteile einer EWU-Mitgliedschaft gesehen. Diese verspricht unter anderem ein niedrigeres Zinsniveau, geringere Risikoprämien, die derzeit Abwertungsrisiken einpreisen, mehr Stabilität durch weniger Wechselkursschwankungen und eine höhere Glaubwürdigkeit für die nationalen Bankensektoren. Insbesondere die Tatsache, dass die Slowakei (EWU-Beitritt am 1. Januar 2009) im zweiten Halbjahr 2008 unter deutlich weniger Druck der Finanzmärkte geriet als andere mittel- und osteuropäische Staaten, hat das Interesse einiger mittel- und osteuropäischer Länder an einer baldigen EWU-Mitgliedschaft verstärkt. ${ }^{18}$

Dies bedeutet allerdings nicht, dass sich die Ost-Erweiterung der Währungsunion tatsächlich beschleunigt. Zwar dämpfen die niedrigen Wachstumsraten und gesunkenen Rohstoffpreise die Inflation in den Kandidatenländern und erleichtern in dieser Hinsicht die Erfüllung der Konvergenzkriterien. Doch gleichzeitig dürften in der aktuellen Krise die Konvergenzerfüllung im Bereich der Fiskalpolitik und der zu beweisenden Wechselkursstabilität schwierig werden.

Eine Aufweichung der seit dem Vertrag von Maastricht unverändert bestehenden Konvergenzkriterien ist aus heutiger Sicht indes unwahrscheinlich. Nicht nur sind sie Bestandteil des EG-Vertrags. Die bisherigen Erfahrungen in zehn Jahren Währungsunion haben überdies aufgezeigt, dass zu viel Heterogenität etwa bei der Entwicklung der Lohnstückkosten und der Inflation wie im Falle Italiens, Portugals oder Griechenlands einigen Mitgliedstaaten hohe Anpassungskosten auferlegt und für politische Spannungen in der Währungsunion sorgt. Eine restriktive Auslegung der Konvergenzkriterien maximiert das Potenzial von Europäischer Kommission und EZB, durch eine zeitliche Verzögerung des EWU-Beitritts oder Präzedenzfälle (wie die Ablehnung der Mitgliedschaft von Litauen im Jahr 2006) den externen ,top-down' Reformdruck auf künftige Mitglieder der Eurozone aufrechtzuerhalten. ${ }^{19}$

\section{Institutionelle Dynamiken in der EWU}

Die ersten zehn Jahre der EWU sind durch ein hohes Maß an ,konstitutioneller' Stabilität gekennzeichnet. Seit der Vertrag von Maastricht die institutionellen Parameter der EWU erstmals festlegte, sind, trotz erfolgter Vertragsrevisionen, ${ }^{20}$ die primärrechtlichen Grundlagen der EWU weitgehend unverändert geblieben. So stellt etwa die EZB nach wie vor den ,,institutionellen Kern“ (,,institutional core“) ${ }^{21}$ ohne primärrechtlich verankertes politisches Gegengewicht auf EWU-Ebene dar und die Aufgabenverteilung zwischen europäischer und nationaler Ebene wurde nicht angetastet. Unterhalb der Primärrechtsebene sind jedoch in den ersten zehn Jahren der EWU institutionelle und politische Dynamiken auszumachen, die es den Mitgliedstaaten der EWU erlaubt haben, einige Governance-Mechanismen den gegebenen Bedingungen anzupassen.

18 The Economist Intelligence Unit: European economy: an unattainable safe haven?, 17.11.2008.

19 Kenneth Dyson: European States and the Euro Area: Clustering and Covariance in Patterns of Change, in: Kenneth Dyson (Hrsg.): The Euro at 10. Europeanization, Power, and Convergence, Oxford 2008, S. 378-413, hier S. 409.

20 Vgl. Vertrag von Amsterdam, in: Amtsblatt der EU, Nr. C 340 vom 10. November 1997; Vertrag von Nizza, in: Amtsblatt der EU, Nr. C 80 vom 10. März 2001. Auch der Vertrag über eine Verfassung für Europa, in: Amtsblatt der EU, Nr. 310 vom 16. Dezember 2004, und der Vertrag von Lissabon, in: Amtsblatt der EU, Nr. C 306 vom 17. Dezember 2007, berühren die institutionellen Rahmenbedingungen der EWU nur unwesentlich (siehe unten). Einen guten Überblick über die moderaten Änderungen geben Gaby Umbach/Wolfgang Wessels: The Changing European Context of Economic and Monetary Union: ,Deepening', ,Widening', and Stability, in: Kenneth Dyson (Hrsg.): The Euro at 10. Europeanization, Power, and Convergence, Oxford 2008, S. 54-68, insbesondere S. 54-57.

21 Ebenda, S. 58. 


\section{Die Reform des Stabilitäts- und Wachstumspakts}

Zweimal seit Verabschiedung des Vertrags von Maastricht wurden bislang die Regeln für die fiskalpolitische Kontrolle in der Währungsunion verändert. ${ }^{22}$ Dies war zunächst die Annahme des Stabilitäts- und Wachstumspakts im Jahr 1997, der noch vor Beginn der Währungsunion auf deutsches Drängen hin die Regeln des Maastrichter Vertrags präzisierte und verschärfte und die Grundlage für ein beschleunigtes Sanktionsverfahren legte.

Der Pakt selbst wurde im Jahr 2005 reformiert, um mehr politischen Ermessensspielraum bei seiner Anwendung zuzulassen, und insbesondere die Berücksichtigung konjunktureller Rahmenbedingungen zu erleichtern. Der Reform vorausgegangen war in den Jahren schwachen Wachstums (2002-2004) ein wiederholter Bruch der Defizitregeln durch Deutschland, Frankreich, Portugal und Italien. Insbesondere unter dem Druck der deutschen und der französischen Regierung entschied der Ecofin-Rat, die Anwendung des Pakts vorläufig auszusetzen, um eine Sanktionierung der Länder, die die Defizitvorgaben überschritten hatten, zu verhindern. Das Urteil des Europäischen Gerichtshofs, den die Europäische Kommission angerufen hatte, erklärte einen Teil der Ecofin-Entscheidung für nicht rechtmäßig, wodurch die ohnehin vorhandenen Bestrebungen, den Pakt zu reformieren, verstärkt wurden.

Die im Jahr 2005 schließlich erfolgte Reform des Pakts sollte dessen ökonomische Rationalität steigern, indem die besonderen konjunkturellen Situationen einzelner Mitgliedstaaten stärker berücksichtigt werden. Zudem wurde der präventive Arm des Pakts gestärkt, also das Verfahren der multilateralen Überwachung und das sogenannte ,Frühwarnsystem', das dabei helfen soll, erhebliche Abweichungen vom mittelfristigen Haushaltsziel zu vermeiden. Diese Reformen sollten die Akzeptanz des Regelwerks durch die Regierungen stärken und gleichzeitig ihren Handlungsspielraum in der Anwendung des Pakts und somit in der Gestaltung nationaler Fiskalpolitik erweitern. Bei alledem ging es darum, die Glaubwürdigkeit des bereits für ,tot" erklärten Regelwerks wieder zu verbessern, die stark gelitten hatte, weil der Pakt gebrochen worden war und Sanktionen gegenüber den größten EWU-Mitgliedern ausgeblieben waren.

Die Reform des Pakts war in eine breitere Diskussion über die Rolle und Kontrolle von nationaler Fiskalpolitik in der Eurozone eingebettet, die bis heute andauert. ${ }^{23}$ Noch im Vorfeld und zu Beginn der EWU war die Frage vorherrschend, wie nationale Fiskalpolitik so kontrolliert werden könnte, dass sie niedrige Defizite und geringe Inflation unterstützen könne. Unter dem Eindruck des Wachstumseinbruchs in der EWU und vor dem Hintergrund neuer Einsichten der internationalen volkswirtschaftlichen Debatte gewann jedoch die Frage an Bedeutung, welche Formen und Ziele der fiskalpolitischen Koordinierung nachhaltiges Wachstum erlauben. Die Diskussion um die Bedeutung der konjunkturellen Stabilisierung durch automatisch wirkende fiskalische Mechanismen gewann im Rahmen der, Wirtschaftspolitischen Leitlinien ' an Raum, also in dem jährlich von den Mitgliedstaaten verabschiedeten Bericht, der das Kernstück der wirtschaftspolitischen Koordinierung in der Europäischen Union bildet. Zudem wurde der Nachhaltigkeit der Staatsfinanzen immer mehr Raum in der Diskussion gegeben, unter anderem, weil sich gezeigt hatte, dass während der Boomphase der Jahre 2000 bis 2002 einige Länder nicht ausreichend konsolidiert hatten. Bereits zur Zeit der Reform des Stabilitäts- und Wachstumspakts wurde überdies gelegentlich über die Rolle

22 Siehe zum Beispiel Daniela Schwarzer: Fiscal Policy Co-ordination in the EMU. A Preference-Based Explanation of Institutional Change, Baden-Baden 2006.

23 Für eine längere Fassung des folgenden Arguments siehe Sebastian Dullien/Daniela Schwarzer: Building on shifting sand: Political Science and the Economic Consensus of EMU, Paper präsentiert bei dem Workshop „10 Years of the European Monetary Union” in der Hertie School of Governance, Berlin 24./25. April 2008. 
diskretionärer Fiskalpolitik diskutiert. Erst unter dem Eindruck der globalen Wirtschaftskrise 2008 jedoch rückten kurzfristige fiskalische Maßnahmen zur Stabilisierung der Konjunktur in den Mittelpunkt der europäischen Debatte.

\section{Lissabon-Agenda und Leitlinien für Wachstum und Beschäftigung}

Die Lissabon-Agenda wurde im Jahr 2005 gestrafft und stärker auf die Förderung von Wachstum und Beschäftigung ausgerichtet. Das Ziel, bis 2010 der wettbewerbsfähigste Wirtschaftsraum zu werden, wurde aufgegeben und die Wachstums- und Beschäftigungsentwicklung wurde in den Mittelpunkt gerückt.

Die ,Grundzüge der Wirtschaftspolitik ' und die ,Leitlinien zur Beschäftigungspolitik ${ }^{\text {‘2 }}$ wurden vom Europäischen Rat im Frühjahr 2005 zu den ,Integrierten Leitlinien für Wachstum und Beschäftigung' zusammengefasst und eng mit der Lissabon-Agenda verzahnt. Seit einiger Zeit wird in den Arbeitsdokumenten die besondere Situation der Eurozone angesprochen. $^{25}$

Wichtiger noch als die Konsolidierung einer Vielzahl zuvor parallel zueinander publizierter Dokumente liegt die Bedeutung dieser Reformen darin, dass ein konsistenterer Koordinierungsprozess geschaffen wurde, der zuvor parallel laufende Politikzyklen in stark interdependenten Feldern, wie zum Beispiel der Lissabon-Agenda und der vormals im Rahmen des Luxemburger Prozesses erfolgenden Koordinierung der nationalen Beschäftigungspolitiken, fusionierte und auf das jährlich stattfindende Frühjahrstreffen des Europäischen Rats hin ausrichtete. Darüber hinaus wurden europäische Koordinierungsprozesse besser mit nationalen Politikzyklen verzahnt. Das Potenzial, über weiche Koordinierung tatsächlich nationale Politiken zu beeinflussen, wurde so leicht gesteigert.

\section{Eurogruppe als wichtigstes Koordinationsforum}

Die wichtigste institutionelle Innovation der Eurozone ist die Eurogruppe. Sie ist ein informelles Gremium der Wirtschafts- und Finanzminister der Eurozonenmitgliedstaaten und stellt bislang das einzige regelmäßig zusammentretende EWU-Forum auf Regierungsebene dar. ${ }^{26}$

Die Eurogruppe wurde bei ihrer Gründung weder im Sekundär- noch im Primärrecht verankert. Sie gehört nicht zum Rat, auch wenn sie sich auf das gleiche vorbereitende Gremium (den Wirtschafts- und Finanzausschuss) stützen kann. Sie hat überdies keine Entscheidungsbefugnisse und kein präzises Mandat und die Interaktion mit der EZB, der Europäischen Kommission und dem Europäischen Parlament ist nicht formal geregelt.

Bestrebungen zur Einführung eines exklusiven Forums für Regierungsvertreter der EWU-Staaten gingen vor allem von Frankreich aus, das sich bereits während der Verhandlungen zum Vertrag von Maastricht für eine stärkere politische Komponente gegenüber der Europäischen Zentralbank eingesetzt hatte. Ende 1996/Anfang 1997, als die Verhandlungen über den von Deutschland vorgeschlagenen Stabilitäts- und Wachstumspakt kurz vor dem Abschluss standen, forderte Frankreich erneut ein wirtschaftspolitisches Gegengewicht zur

24 Vertragsgrundlage für die Koordinierung in beiden Feldern sind Art. 99 und Art. 128 EG-Vertrag.

25 Siehe beispielsweise: Europäische Kommission: The broad economic policy guidelines (for the 2005-08 period), in: European Economy 4/2005, insbesondere S. 15-16.

26 Der Makroökonomische Dialog (auch Kölner Prozess genannt) bringt seinerseits Regierungsvertreter der Eurozone gemeinsam mit den Sozialpartnern sowie Vertretern von Kommission und EZB in zwei jährlichen Sitzungen zusammen. Allerdings finden diese Sitzungen nur einmal im Jahr auf politischer Ebene statt (die zweite Sitzung ist auf technischer Ebene). Der Austausch ist aufgrund der größeren Teilnehmerzahl und der geringeren Frequenz der Treffen aber weniger kontinuierlich und eng als in der Eurogruppe. Zum Kölner Prozess im Allgemeinen siehe Torsten Niechoj: Koordinierung à la Keynes? Der Makroökonomische Dialog des Kölner Prozesses, in: integration 1/2005, S. 68-80. 
EZB im von deutscher Seite maßgeblich gestalteten ordoliberalen Modell der EWU. Traditionell bestehen zwischen den beiden größten EU-Staaten und Motoren hinter der Gemeinschaftswährung, Deutschland und Frankreich, divergierende Ansichten über eine geeignete Ausgestaltung der Währungsunion, insbesondere über die Rolle der EZB und einer möglichen Wirtschaftsregierung. ${ }^{27}$ Diese Divergenzen wurden mit dem Kompromiss im Vertrag von Maastricht nicht beigelegt, sondern treten auch heute immer wieder zutage.

Neben Frankreich waren auch andere Beteiligte und Beobachter nach den ersten Erfahrungen mit dem Vertrag von Maastricht zur Auffassung gekommen, dass die im Vertrag festgelegten Institutionen und Prozesse für die wirtschaftspolitische Koordinierung in einem gemeinsamen Währungsraum möglicherweise nicht ausreichen. ${ }^{28}$ Beim informellen Ecofin-Treffen im luxemburgischen Mondorf-les-Bains im September 1997 und beim deutsch-französischen Wirtschaftsrat am 14. Oktober 1997 kristallisierte sich ein möglicher Kompromiss heraus: ein informeller Kreis der Wirtschafts- und Finanzminister mit eindeutig abgesteckter Aufgabe, da Deutschland keine institutionalisierte Wirtschaftsregierung akzeptierte, um - auch mit Blick auf die nationale Diskussion um den baldigen Beginn der EWU - jedweden Zweifel an der institutionellen und politischen Unabhängigkeit der EZB zu verhindern. ${ }^{29}$ Die Einführung der Eurogruppe geht auf einen Beschluss des Europäischen Rats am 13. Dezember 1997 in Luxemburg zurück, der für die Minister der EWU-Staaten die Möglichkeit schuf, ,in informellem Rahmen Fragen [zu] erörtern, die im Zusammenhang mit ihrer gemeinsam getragenen besonderen Verantwortung für die gemeinsame Währung stehen“ ${ }^{30}$ Die Kommission, und gegebenenfalls die EZB, sollen zu diesen Zusammenkünften eingeladen werden. Dies brachte die Unterstützung insbesondere der kleineren Staaten, die darin die Chance auf einen Dialog mit der EZB sahen.

Für die Ausgestaltung der Arbeit der Eurogruppe war die Interaktion und letztendlich auch die Zustimmung des Ecofin-Rats und des Europäischen Rats entscheidend. Die Schlussfolgerungen einiger Europäischer Räte haben seit 1998 Teilaspekte geregelt, etwa die externe Vertretung der Eurozone, die Rolle der Eurozone in der internen Koordinierung der Wirtschaftspolitiken in der EWU sowie die Einführung eines Eurogruppenpräsidenten. Für die EWU-Staaten wurde mit jedem weiteren Jahr der Währungsunion die Bedeutung der Eurogruppe für die Vertrauensbildung, den Meinungs- und Informationsaustausch und die Formulierung von gemeinsamen Positionen deutlicher. Die schwierige Wirtschaftslage der Jahre 2002/2003 und die politischen Spannungen über die Anwendung des Stabilitäts- und Wachstumspakts machten die Bedeutung des Gremiums für die Diskussionen über die Inhalte und Verfahren der fiskal- und wirtschaftspolitischen Koordinierung deutlich.

Die bislang umfassendste Reform erfolgte am 10. September 2004, als die Eurogruppe durch institutionelle und inhaltliche Neuerungen gestärkt wurde. Die Vereinbarung darüber wurde bei einem informellen Treffen der Wirtschafts- und Finanzminister der Eurozone im niederländischen Scheveningen getroffen. ${ }^{31}$ Die Eurogruppe bekam einen zweijährigen Vor-

27 Siehe zum Beispiel Jean Pisani-Ferry: Only One Bed for Two Dreams: A Critical Retrospective on the Debate over the Economic Governance of the Euro Area, in: Journal of Common Market Studies 4/2006, S. 823-844.

28 Uwe Pütter: The Eurogroup. How a secretive circle of finance ministers shape European economic governance, Manchester 2006, S. 58.

29 Stefan Collignon/Daniela Schwarzer: Private Sector Involvement in the Euro. The power of ideas, London 2003, S. 159-170.

30 Entschließung des Europäischen Rates vom 13. Dezember 1997 über die wirtschaftspolitische Koordinierung in der dritten Stufe der WWU und zu den Artikeln 109 und 109b des EG-Vertrags, in: Amtsblatt der EU, Nr. C 35 vom 2. Februar 1998, S. 1-4.

31 Siehe hierzu: Bundesfinanzministerium: Europäische Wirtschafts- und Finanzpolitik. Stärkung der Eurogruppe, in: Monatsbericht des BMF 9/2004, S. 26-27; Europa-Informationen: Eurogroup ministers agree on new working methods - Jucker appointed as Eurogroup president, 10.09.2004. 
sitz, der die Außenvertretung der Eurozone stärken und die wirtschafts- und finanzpolitischen Weichenstellungen kohärenter und klarer kommunizierbar machen sollte. ${ }^{32}$ Die Tagesordnung der Eurogruppensitzungen sollte strategischer ausgerichtet und mehr Zeit auf die Diskussion makro- und strukturpolitischer Themen verwendet werden. Ein wichtiger Schwerpunkt der Diskussion soll die Haushaltspolitik werden, indem Anfang Juni jedes Jahres eine Orientierungsaussprache über die Haushaltsplanungen geführt wird, bevor Haushaltsbeschlüsse in den Mitgliedstaaten getroffen werden. Ziel ist es, bessere Einsichten darüber zu gewinnen, wie sich die nationale Politik in die generelle Ausrichtung der Finanzpolitik in der Eurozone einfügt. Die Diskussion über die jeweiligen nationalen Wachstumsprognosen soll überdies offener geführt werden, um ein möglichst klares Verständnis über die wirtschaftlichen Aussichten der Union zu erhalten.

\section{Gründe für die informelle Natur der Eurogruppe}

Der Charakter der Eurogruppe trägt der Tatsache Rechnung, dass die institutionellen Mechanismen der Europäischen Union und der EWU auf jeweils unterschiedliche Teilnehmerländer ausgelegt sein müssen: Während die EZB mit ihrer Geldpolitik per Definition nur für die derzeit 16 Währungsunionsländer zuständig ist, umfasst der Ecofin-Rat als das entscheidende Organ in der Wirtschaftspolitik der Europäischen Union alle 27 EU-Mitglieder.

Die angesprochenen Regelungen des Maastrichter Vertrags zum Ecofin-Rat und den wirtschaftspolitischen Entscheidungsmechanismen wurden mit dem Ziel gestaltet, die institutionelle Einheit des Ecofin-Rates bei einer zeitlich begrenzten nicht identischen Mitgliedschaft zwischen Europäischer Union und EWU aufrechtzuerhalten. Um im Rahmen des Ecofin-Rates EWU-relevante Entscheidungen durch EWU-Mitgliedstaaten fällen zu lassen, wurden abgestufte Stimmrechte vorgesehen. ${ }^{33}$ Durch die Entscheidung Dänemarks, Großbritanniens und später auch Schwedens, sich eine Nichtteilnahme an der EWU vorzubehalten, wurde der Ecofin-Rat jedoch als Forum für wirtschaftspolitische Diskussionen und Entscheidungen mit Relevanz für den gemeinsamen Währungsraum ungeeignet. Durch den Beitritt von zehn neuen Ländern zur Europäischen Union am 1. Mai 2004 verstärkte sich überdies die Notwendigkeit eines exklusiven Forums für die EWU-Mitglieder, zumal sich der Konvergenzprozess schleppender als ursprünglich angenommen gestaltet.

Der informelle Charakter der Eurogruppe ist ein Zugeständnis an die Opt-Out-Staaten und Noch-Nicht-Mitglieder, die eine institutionelle und politische Abkopplung der Eurozone von der Europäischen Union insgesamt verhindern wollten. Zudem trägt er den widerstreitenden Konzepten zur Gestaltung der Eurozone Rechnung, da für eine Institutionalisierung eines exklusiven Eurozonenforums im EG-Vertrag keine Mehrheit zu gewinnen war.

Der am 13. Dezember 2007 unterzeichnete Vertrag von Lissabon gibt - im Falle seiner erfolgreichen Ratifizierung - der Eurogruppe erstmals eine primärrechtliche Grundlage. Das entsprechende Protokoll ${ }^{34}$ kodifiziert die bestehende Praxis, dass die Minister der EWUStaaten zu informellen Sitzungen zusammentreten. Die Kommission nimmt teil, die EZB wird eingeladen. Darüber hinaus sieht das Protokoll die Wahl eines Eurogruppenpräsidenten für einen Zeitraum von zweieinhalb Jahren vor.

32 Weder die Vereinbarung, noch das darauf beruhende Protokoll zum Europäischen Verfassungsvertrag, legen dabei fest, dass der Vorsitzende aus der Mitte des Gremiums stammen muss. So ist es zum Beispiel denkbar (und wurde im Verfassungskonvent auch so vorgeschlagen), dass ein Mitglied der Europäischen Kommission dieses Amt übernimmt.

33 Die sogenannten,Outs ' haben bei Art. 104 Abs. 9, 104 Abs. 11, 106 Abs. 2, 111, 112 Abs. 2b, 123 Abs. 4 und 123 Abs. 5 EG-Vertrag kein Stimmrecht, also bei der Wechselkurspolitik, bei der Besetzung von EZB-Posten ebenso wie bei bestimmten Implementierungsschritten des Stabilitäts- und Wachstumspakts. 


\section{Schrittweise Anpassung der Regeln und Gremien}

Maßgebliche Faktoren für die schrittweise Etablierung und schließlich die Kodifizierung der Eurogruppe und ihrer besonderen Funktionsweise waren die Erfahrung der Teilnehmer mit dem Gremium und den weiteren Politikkoordinierungsprozessen in der EWU ${ }^{35}$ sowie realwirtschaftliche Entwicklungen und daraus resultierende Kooperationsnotwendigkeiten.

$\mathrm{Zu}$ Zeiten des Verfassungskonvents hatte sich in der Eurogruppe bereits ein stabiler Modus Vivendi herausgebildet, der durch die interne Reform des Gremiums im Jahr 2004 bestätigt und weiterentwickelt wurde. Sehr deutlich war der Bedarf und das Interesse an einer Abstimmung mit den traditionellen EU-Institutionen (EZB, Kommission, Ecofin-Rat, Europäisches Parlament) geworden. So formulierten etwa Abgeordnete des Europäischen Parlaments im Rahmen des Verfassungskonvents sehr klare Vorstellungen zur Zusammenarbeit mit der Eurogruppe. Zudem ging es den EWU-Staaten auch darum, die Eurogruppe vor der EU-Erweiterung um zehn neue Mitgliedstaaten formal abzusichern, da mit der Neumitgliedschaft das zahlenmäßige Ungleichgewicht zwischen EWU-Mitgliedern und EWU-Nichtmitgliedern von 12 zu 15 auf 12 zu 25 wuchs.

Das angespannte konjunkturelle Umfeld der Jahre 2002/2003 beschleunigte nicht nur die Reform der Eurogruppe, sondern warf die darüber hinausgehende Frage nach den Verfahren und Inhalten der wirtschaftspolitischen Koordinierung in der Eurozone mit neuer Dringlichkeit auf. Hinzu kamen die ersten praktischen Erfahrungen mit der Anwendung des im Maastrichter Vertrag vorgesehenen Verfahrens- und Regelwerks. Vor diesem Hintergrund sind die Reform des Stabilitäts- und Wachstumspakts ebenso wie die Refokussierung und das Streamlining der anderen Koordinationsprozesse zu sehen.

\section{Krisenmanagement und Neuerungen 2008}

Eine besondere Herausforderung erlebte die Eurozone im zweiten Halbjahr 2008, als drohende Zusammenbrüche im Finanzsektor, der massive Konjunktureinbruch und die mögliche Zahlungsunfähigkeit einiger EWU-Nachbarstaaten ökonomische wie politische Spannungen und Instabilitäten ankündigten. Unter dem Druck der Krise erfolgte eine flexible Anpassung einiger Politiken und Governance-Mechanismen.

\section{Erster Eurozonen-Gipfel}

Der zum 12. Oktober 2008 vom amtierenden EU-Ratspräsidenten Nicolas Sarkozy einberufene erste Eurozonen-Gipfel war ein historisches Novum. Nicht nur trafen sich die damals 15 EWU-Staaten (sowie Großbritannien) erstmals auf Ebene der Staats- und Regierungschefs um Eurozonen-Belange zu besprechen. Sie einigten sich überdies auf einen gemeinsamen Plan zur Rettung des Bankensystems (Gewährung von Staatsgarantien für mittelfristige Schuldtitel und die Möglichkeit von Staatsbeteiligungen an Kreditinstituten). Zudem wurde vereinbart, die nationalen Rettungspläne zu koordinieren und die Arbeit an besseren Aufsichtsstrukturen für die Finanzmärkte zu beschleunigen.

34 Protokoll Nr. 14 betreffend die Euro-Gruppe, beigefügt den konsolidierten Fassungen des Vertrags über die Europäische Union und des Vertrags über die Arbeitsweise der Europäischen Union in der Version des Vertrags von Lissabon, in: Amtsblatt der EU, Nr. C 115 vom 9. Mai 2008, S. 283. Dieses Protokoll entspricht dem ursprünglich dem Vertrag über eine Verfassung für Europa beigefügten 12. Protokoll betreffend die EuroGruppe, in: Amtsblatt der EU, Nr. C 310 vom 16. Dezember 2004, S. 341.

35 Etwa der fiskalpolitischen Koordinierung im Rahmen des Stabilitäts- und Wachstumspakt, der darüber hinausgehenden wirtschaftspolitischen Abstimmung im Rahmen der , Wirtschaftspolitischen Leitlinien " oder auch der Zusammenarbeit im Rahmen des Makroökomischen Dialogs („Kölner Prozess“). 
Vorausgegangen waren deutsch-französische Auseinandersetzungen um die Notwendigkeit einer verstärkten politischen Koordinierung, deren Grundargumente so alt sind wie die Debatte um die Einführung einer Gemeinschaftswährung in der Europäischen Union. Die französische Regierung und Staatspräsident Sarkozy hatten die Weiterentwicklung der EWU zunächst als eine mögliche Priorität ihrer EU-Ratspräsidentschaft im 2. Halbjahr 2008 in Erwägung gezogen und immer wieder die Einführung eines ,gouvernement économique“ gefordert. Konkrete Initiativen blieben jedoch zunächst aus. Premierminister François Fillon warb zwar im Januar 2008 dafür, die Staats- und Regierungschefs zu einem Eurozonen-Gipfel zu versammeln. Dieser sollte sich nach Vorlage eines Berichts der Europäischen Kommission zur Funktionsweise der Eurozone im ersten Halbjahr 2008 noch vor Beginn oder während der französischen EU-Ratspräsidentschaft mit Fragen der wirtschaftspolitischen Koordinierung und der Außenvertretung des Euro beschäftigen. ${ }^{36}$ Doch insbesondere die abwehrende Haltung der Bundesregierung führte dazu, dass dieses Thema zunächst nicht weiter verfolgt wurde.

Mit der Zuspitzung der internationalen Finanzkrise im Herbst 2008 initiierte der amtierende EU-Ratspräsident Sarkozy jedoch eine Serie an unterschiedlichen Gipfelformaten, darunter den ersten Eurozonen-Gipfel. Wiederum sorgten deutsch-französische Auffassungsunterschiede zunächst für Spannungen, etwa beim vorangehenden G4-Treffen, an dem neben Deutschland und Frankreich Großbritannien, Italien und Kommissionspräsident Barroso sowie EZB-Präsident Jean-Claude Trichet teilnahmen. Deutschland lehnte dabei vehement die vorgeschlagene Schaffung eines europäischen Bankenauffangfonds ab und reagierte gewohnt ablehnend auf Vorschläge, die „gouvernance“ der Eurozone zu verstärken.

\section{Geldpolitische Reaktion und neuer fiskalpolitischer Kurs}

Die EZB stellte sich in der Finanzkrise rasch auf ihre Rolle als ,Lender of last Resort“ ein. Hierzu gehörte unter anderem auch eine Anpassung der Instrumente (so etwa des Timings für die Liquiditätsbereitstellung). ${ }^{37}$ Sie ging darüber hinaus internationale Kooperationen ein, so beispielsweise im Oktober 2008 mit der US-Notenbank.

Nachdem sich die Wirksamkeit der geldpolitischen Maßnahmen zur Abfederung der Krise als begrenzt erwiesen hatte, bildete sich schrittweise - explizit gefordert von der EZB und dem Internationalen Währungsfonds (IWF) - ein Konsens heraus, dass die Europäische Union und die mitgliedstaatlichen Regierungen auch finanzpolitisch auf die Krise reagieren müssten.

Da zunächst nur eine Reihe nationaler Maßnahmen nebeneinander standen, unternahm die Europäische Kommission den Versuch, nationale Konjunkturstützungspakete EU-weit zu koordinieren und auf europäischer Ebene zu flankieren. Dieser Ansatz trägt der Tatsache Rechnung, dass bei offenen Volkswirtschaften Nachfragestimulierung nicht mehr sinnvoll im nationalen Rahmen vorgenommen werden kann, weil die Impulse über die integrierten Märkte sofort in den weiteren Wirtschaftsraum weitergereicht werden. Ein aus ökonomischer Sicht sinnvoller Rahmen für eine nachfrageorientierte Fiskalpolitik ist demnach insbesondere die Eurozone mit einem integrierten Geldwesen, stark integrierten Märkten und einem mit 22,6 Prozent des BIP vergleichsweise geringen Offenheitsgrad. ${ }^{38}$

36 Wolfgang Proissl/Peter Ehrlich: Sarkozy wagt neuen Anlauf für EU-,,Wirtschaftsregierung“, in: Financial Times Deutschland, 15.01.2008.

37 Werner Becker: Die Währungsunion im Reifetest der Finanzkrise, in: Deutsche Bank Research (Hrsg.): Aktueller Kommentar, 29.10.2008.

38 Europäische Zentralbank: Monthly Bulletin. 10th Anniversary of the ECB. 1998-2008, Frankfurt am Main 2008, S. 152. 
Jahrelang hatte sich etwa die Kommission gegen diskretionäre Konjunkturstimulierung ausgesprochen und im Zuge der Überwachung der Haushaltspolitik der EU-Mitgliedstaaten entsprechende nationale Budgetentscheidungen kritisiert. Nun ist diskretionäre Haushaltspolitik als Ausweg aus der Krise anerkannt - wenngleich es in der Währungsunion keinen Konsens über die ,richtige‘ Lastenteilung gibt. So wurde beispielsweise die Bundesregierung von EU-Partnern, der Europäischen Kommission und dem IWF im Herbst 2008 aufgefordert, vor dem Hintergrund einer sehr soliden Haushaltslage die Konjunktur durch fiskalische Maßnahmen noch stärker zu stimulieren, da von einer Erholung der deutschen Volkswirtschaft wichtige Wachstumsimpulse für den gesamten Euroraum erwartet werden.

An den Divergenzen zwischen der deutschen Position und den Forderungen der EU-Partner lassen sich zwei strukturelle Probleme aufzeigen: Zum einem gibt es auch heute (insbesondere zwischen Deutschland und Frankreich) keinen wirtschaftspolitischen Konsens, in diesem Fall über die Frage, welches Maß an fiskalischer Stabilisierung angemessen wäre. Aufgrund der nationalen Zuständigkeit für die Budgetpolitik und aufgrund des Volumens und der Struktur des EU-Budgets ist der europäische Handlungsspielraum im Falle eines Dissenses der Regierungen gering. Zum zweiten gibt es im gemeinsamen ,Wirtschaftsraum Währungsunion' angesichts der offenen Grenzen bei den derzeitigen Strukturen eine Diskrepanz zwischen demjenigen, der die Kosten trägt (im Falle Deutschlands der Bundeshaushalt) und denjenigen, die in den ,Genuss' der Maßnahmen kommen (aufgrund des Offenheitsgrades der Märkte auch Deutschlands EU-Partner). Dieses Problem wird aus deutscher Sicht tendenziell so gesehen, dass die Bundesregierung nicht, für die anderen zahlen will'. Aus Sicht mancher Partner jedoch verhält sich gerade Deutschland mit seiner fiskalpolitischen Zurückhaltung bei einer soliden finanziellen Haushaltslage als , Trittbrettfahrer', der seiner Verantwortung in der Europäischen Währungsunion nicht gerecht werde und gleichsam von den Konjunkturprogrammen der anderen EWU-Staaten aufgrund deren Nachfrage nach deutschen Exportgütern mitprofitiere. ${ }^{39}$

\section{Weitere Auswirkungen auf die Governance-Mechanismen}

Die Notwendigkeit, die europäische Volkswirtschaft in der Rezession fiskalisch zu stimulieren, hat möglicherweise weitere Auswirkungen auf die Governance-Mechanismen der Eurozone. So wird aller Voraussicht nach der Stabilitäts- und Wachstumspakt zunächst einmal locker angewendet: Bislang haben sich die Europäische Kommission, ebenso wie die beiden größten Eurozonenländer Deutschland und Frankreich ${ }^{40}$ dafür ausgesprochen, EUStaaten die Möglichkeit einzuräumen, aufgrund der notwendig gewordenen konjunkturellen Stabilisierung die Defizitobergrenze von drei Prozent des BIP zu überschreiten, ohne dass diese Sanktionen fürchten müssen. Bei mehr kurzfristiger Diskretion für die nationale Fiskalpolitik dürfte allerdings, durch die Europäische Kommission und einige Mitgliedstaaten wie Deutschland und auch die Niederlande angetrieben, das Thema der Qualität der Staatsfinanzen und der langfristigen Nachhaltigkeit wieder stärker auf die europäische Agenda drängen. Möglicherweise wird der Pakt auch einer erneuten Reform unterzogen, sollten sich die Spielräume für die nationale Fiskalpolitik, auf konjunkturelle Schwankungen zu reagieren, als immer noch zu gering erweisen.

39 Siehe zum Beispiel Wolfgang Münchau: German complacency poses a serious threat, in: Financial Times, 30.11.2008.

40 Angela Merkel/Nicolas Sarkozy: „Nous ne pouvons pas attendre“, in: Le Figaro, 26.11.2008. 


\section{Möglicher Stabilisierungsbedarf}

Es ist zudem nicht auszuschließen, dass wachsender Druck durch die Finanzmärkte den Stabilisierungsbedarf von EU-Partnern, die noch nicht Mitglied der Währungsunion sind, erhöht. In diesem Fall würde der Kapitalbedarf der Gemeinschaft erheblich wachsen.

Auf Grundlage von Artikel 119 EG-Vertrag ${ }^{41}$ hat der Ecofin-Rat Ungarn am 4. November 2008 einen dreijährigen Kredit in Höhe von 6,5 Milliarden Euro gewährt, um die Zahlungsbilanzschwierigkeiten der Staaten abzumildern und den Zusammenbruch der jeweiligen Finanzsektoren zu verhindern. Auf gleicher Grundlage dürfte auch Lettland finanzieller Beistand gewährt werden. Um diese Maßnahmen zur Stabilisierung der Peripherie der Eurozone zu bezahlen, legt die Europäische Kommission zweckgebundene Euro-Bonds (oder auch EU-Staatsanleihen) auf, um den Empfängerländern ,Balance-of-Payment-Loans` zu gewähren, die seit den 1990er Jahren nicht mehr zum Einsatz kamen. Diese Finanzierungsmethode war zunächst bei 12 Milliarden Euro gedeckelt, wurde allerdings im Dezember 2008 durch einen Beschluss des Ecofin-Rats auf Vorschlag der Europäischen Kommission auf 25 Milliarden Euro erhöht.

Ein Grund, warum der Stützungsbedarf plötzlich so hoch eingeschätzt wird, könnte sein, dass ernsthafte Risiken darin gesehen werden, dass die Finanzsektoren in EU-Staaten und damit im direkten Umfeld der Eurozone zusammenbrechen könnten. Da in der Europäischen Union beziehungsweise EWU angesiedelte Banken in diesen Ländern den Löwenanteil des Finanzsektors stellen, würden dortige Bankenpleiten unmittelbar in die Währungsunion hineinwirken. Eurogruppenpräsident Jean-Claude Juncker hat darüber hinaus gefordert, der Europäischen Union die Möglichkeit einzuräumen, sich an den Finanzmärkten zu verschulden, um Investitionen in Infrastrukturprojekte zu finanzieren. Die aktuelle Krise nährt also - parallel zur ohnehin laufenden Überprüfung des EU-Budgets - die Diskussion um die Finanzierung von EU-Politiken, um die dafür nötige Autonomie der Gemeinschaft und auch um die Frage, inwieweit eine Verschuldung in bestimmten Situationen für die Europäische Union sinnvoll ist.

\section{Künftige Herausforderungen für die Eurozone}

Die kommenden Osterweiterungen der Eurozone werden die Governance-Mechanismen vor weitere Herausforderungen stellen. Zum einen dürfte sich mit der schieren Mitgliederzahl die Atmosphäre und Arbeitsweise der Eurogruppe weiter ändern. Mit 20 und mehr Mitgliedern wird sich der persönlich-vertrauliche Charakter und ihre Fähigkeit zur Konsensbildung abschwächen. Dies gilt umso mehr als mit den künftigen mittel- und osteuropäischen Partnern EU-Staaten zur EWU stoßen werden, die ihre nationalen Präferenzen vor dem Hintergrund einer anderen strukturellen und konjunkturellen wirtschaftlichen Situation formulieren und die überdies in Einzelfällen eine abwartende bis skeptische Haltung zu europäischen Koordinationsprozessen haben.

Auch die künftige Anwendung des Stabilitäts- und Wachstumspakts wird in einer erweiterten Währungsunion als potenziell problematisch gesehen: So erwartet Begg angesichts der andauernden Unterschiede (einige schnell wachsende und sich rasch restrukturierende mittel- und osteuropäische Staaten und langsam wachsende ,alte' Mitglieder der EWU), dass auch die Flexibilität des reformierten Pakts nicht ausreichen könnte, individualisierte

41 Präzisiert durch Verordnung (EG) Nr. 332/2002 des Rates vom 18. Februar 2002 zur Einführung einer Fazilität des mittelfristigen finanziellen Beistands zur Stützung der Zahlungsbilanzen der Mitgliedstaaten, in: Amtsblatt der EU, Nr. L 53 vom 23. Februar 2002, S. 1-3. 
Zielvorgaben schwer umsetzbar sein und die fiskalische Disziplin untergraben könnten. ${ }^{42}$ Die aktuelle Krise dürfte diese Tendenz verschärfen.

Wachsende ökonomische Divergenzen und daraus resultierende divergierende politische Präferenzen hinsichtlich der EWU und ihrer Governance-Mechanismen sind nicht allein ein Ergebnis der Krise, wenngleich diese sie verstärkt. Eine grundlegende Antwort auf die zyklischen Divergenzen in der Währungsunion, die auch ohne Finanz- und Wirtschaftskrise für Spannungen bis hin zu Austrittsdiskussionen gesorgt haben, steht aus. Es bleibt zu beantworten, inwieweit die aktuelle Krise die Tendenz verschärft, dass über die bestehenden Marktmechanismen regional divergierende Entwicklungen nicht ausgeglichen werden können, und - wie in anderen heterogenen Volkswirtschaften - auf nicht-diskretionäre fiskalische Mechanismen zurückgegriffen wird.

Die Wucht, mit der die Krise einige EU-Staaten trifft, könnte im schlimmsten Fall dazu führen, dass auch EWU-Mitglieder, wie Italien, Griechenland oder auch Portugal, durch spekulativen Druck auf die Staatsanleihen in Zahlungsschwierigkeiten geraten. Tritt dieser Fall ein, dürfte die No-Bail-Out-Regel im EG-Vertrag, nach der weder die Europäische Union noch die anderen Mitgliedstaaten für die Schulden eines Mitgliedstaats haften, de facto fallen. Die bestehenden EU-Rechtsgrundlagen sehen vor, dass Nothilfe bei Zahlungsschwierigkeiten nur Nicht-EWU-Staaten gewährt werden kann. Politisch erscheint es nicht akzeptabel, Nicht-EWU-Mitglieder vor der Zahlungsunfähigkeit zu retten, Mitglieder im Euro jedoch nicht in ähnlicher Art und Weise unter die Arme zu greifen, insbesondere, da eine Destabilisierung des Bankensektors massive Spill-over-Effekte in den Rest der Eurozone hätte.

\section{Bedeutung des Lissabon-Vertrags}

Schließlich könnte die mögliche Implementierung des Lissabonner Vertrags weitere institutionelle Dynamiken auslösen. Der Vertrag würde der Eurogruppe eine politisch stärker belastbare Grundlage geben, um ihre Rolle in der Eurozone und innerhalb des Institutionengefüges der Europäischen Union sowie in der Außenvertretung weiter zu stärken. Hinzu kommen zwei Neuerungen: Die Kommission, die offiziell mit der Vorbereitung der Sitzungen beauftragt wird, hat mit dem Protokoll eine Bezugsgrundlage, auf der sie die ihr im EGVertrag zugeschriebene Rolle im Bereich der Wirtschafts- und Währungsunion auch in der Eurozone auf Grundlage eines Konsenses der Mitgliedstaaten ausüben kann. Zweitens sieht das Protokoll vor, die Amtszeit des Präsidenten auf zweieinhalb Jahre zu verlängern. So wird nicht nur die Kontinuität der Arbeit in der Eurogruppe um ein Viertel der bisherigen Amtszeit ausgedehnt. Die längere Amtszeit könnte auch dafür genutzt werden, die fünfjährige Wahlperiode des Europäischen Parlaments und die Präsidentschaft der Eurogruppe zu synchronisieren.

Der Europäische Rat würde auf Grundlage des Lissabonner Vertrags künftig bei der Bewertung der mitgliedstaatlichen Wirtschaftspolitik vor dem Hintergrund der , Wirtschaftspolitischen Leitlinien " mit qualifizierter Mehrheit entscheiden und sich somit über die Stimme des betroffenen Mitgliedstaates hinwegsetzen können. Überdies bestünde für die EWUStaaten die Möglichkeit, mit qualifizierter Mehrheit darüber zu entscheiden, die Überwachung der Haushaltsdisziplin zu verstärken und für EWU-Staaten spezielle Grundzüge der Wirtschaftspolitik auszuarbeiten, die mit den für die gesamte Union angenommenen Grundzügen der Wirtschaftspolitik vereinbar sein müssten.

42 Iain Begg: Economic governance in an enlarged euro area, in: Europäische Kommission (Hrsg.): Economic Papers 311/2008. 
Durch die neue Konzeption der EU-Ratspräsidentschaft würden zudem folgende Funktionsträger im Bereich der Wirtschafts- und Fiskalpolitik zusammenspielen: der neue Präsident des Europäischen Rats, die Dreier-Teampräsidentschaft und der neue Präsident der Eurogruppe. Überdies würde die Rolle der Kommission in der Implementierung des Stabilitätsund Wachstumspakts gestärkt, was die Anreize zur frühzeitigen Koordination zwischen Mitgliedstaaten und Kommission stärkt. ${ }^{43}$

Jenseits dieser Neuerungen wird die künftige Bedeutung der Eurogruppe allerdings wie auch in der Vergangenheit entscheidend davon bestimmt werden, wie die Teilnehmerländer und insbesondere die Präsidentschaft des Gremiums ihre Aufgabe in der Praxis wahrnehmen und wie sich die Beziehungen zu den bereits bisher relevanten institutionellen Partnern EZB, Kommission, Ecofin-Rat und zunehmend auch das Europäische Parlament gestalten.

Ungeachtet der Frage, ob der Lissabonner Vertrag kommt, oder nicht, stehen die Mitgliedstaaten von EWU und Europäischer Union vor der Herausforderung, eine gemeinsame Position in der internationalen Diskussion um künftige Wechselkursregime und die Reform des globalen Wirtschafts- und Finanzsystems zu entwickeln. Offen ist auch, wer die Eurozone künftig nach außen in einer Art vertritt, dass er von den USA, China und Japan als gleichwertiger Partner anerkannt wird. Damit in Zusammenhang steht auch die Frage, ob die EWU künftig ihre Stimmen in wichtigen Foren, wie IWF, G20 etc., zusammenlegt, um als geeinter Akteur aufzutreten.

In den zehn Jahren seit ihrer Gründung haben die Mitgliedstaaten der Währungsunion einige der internen Governance-Regeln und -Mechanismen schrittweise angepasst. Insbesondere die bisherige Reaktion auf die aktuelle Finanzkrise hat gezeigt, dass die Gemeinschaft trotz der komplexen Entscheidungsmechanismen in der Lage ist, flexibel auf die Herausforderungen zu reagieren. Die Krise hat die Bereitschaft zur Koordination und Zusammenarbeit innerhalb der Europäischen Union gesteigert. Sie hat allerdings auch aufgezeigt, dass es keine konsequente Verarbeitung der ökonomischen Interdependenzen in europäisches politisches Handeln gibt. Die zweitgrößte Volkswirtschaft der Welt verfügt trotz der Dynamiken seit Einführung des Euro noch nicht über das Instrumentarium einer einheitlichen Volkswirtschaft, was zu wirtschaftspolitischer Inkohärenz führen kann. Am Beispiel des bislang nicht eingerichteten europäischen Bankenauffangfonds und der nur bedingt vorhandenen Bereitschaft zur Koordinierung der Nachfragestimulierung zeigt sich, dass die Bewertung von Kosten und die Bereitschaft zur Aufteilung von Risiken trotz der starken Interdependenzen weiter vor allem entlang nationaler Grenzen verläuft.

43 Die Kommission kann nunmehr im Falle übermäßiger Defizite einen ,Vorschlag ' statt bisher einer ,Empfehlung ' zur Korrektur der Haushaltspolitik an den Mitgliedstaat abgeben. Einen, Vorschlag ' kann der Ecofin-Rat nur einstimmig (statt bisher mit qualifizierter Mehrheit) abändern. 